\title{
Impact of Prematuration Culture on Zygote Morphology in In Vitro Maturation: An Early Clue to Embryo Competence
}

\author{
Mohammed A Cheruveetil ${ }^{1}$, Prasanna K Shetty ${ }^{2}$, Kamini A Rao ${ }^{3}$, Mir Jaffar ${ }^{4}$, Arya Rajendran ${ }^{5}$, Muhammed Asif $^{6}$, Sumi Maria $^{7}$
}

\begin{abstract}
Background: The study was undertaken to gain insight into the morphology of pronuclear oocytes developed through prematuration culture with cilostamide. The criteria such as position and orientation of pronuclei (PN), the size and distribution of nucleolar precursor bodies (NPB), alignment of polar body (PB), and the cytoplasmic halo have been included in the study.

Objective: To elucidate the effect of cilostamide on zygote morphology in prematuration culture.

Study design: A prospective analysis of pronuclear zygote morphology developed through IVM with prematuration culture using cilostamide conducted from April 2018 to March 2020.

Materials and methods: The present study comprised of 57 zygotes, obtained from the in vitro matured oocytes of 63 patients aged between 25 and 35 years, who underwent controlled ovarian stimulation for IVF/ICSI.

Results: In pronuclear morphology, the central juxtaposed position was higher in the experimental group (77.1\%) than in the control group (54.5\%). The Group 1 NPB distribution was statistically significant in the experimental group (51.4\%) as compared to the control group (22.7\%) $(p=0.03)$. Besides, Group 3 NPBs were lower in the experimental group (11.4\%) when compared to the control group $(36.4 \%)(p=0.02)$. The a-type polar body distribution was significantly higher in the experimental group $(65.7 \%)(p=0.03)$. Cytoplasmic halo was noted in the experimental $(60 \%)$ and control $(45.5 \%)$ groups $(p>0.05)$.

Conclusion: The results indicate that prematuration culture using cilostamide for synchronizing nuclear and cytoplasmic maturation yielded a better pronuclear zygote morphology.

Keywords: Cilostamide, Nucleolar precursor bodies, Polar body, Prematuration culture, Pronuclear zygote, Pronuclei.

International Journal of Infertility and Fetal Medicine (2022): 10.5005/jp-journals-10016-1251
\end{abstract}

\section{INTRODUCTION}

The quest for safer techniques and newer technologies in assisted reproduction has been an enduring concern for researchers globally. In vitro maturation (IVM) of oocytes has been a technique introduced way before the conventional IVF, yet the implantation rates and embryo quality associated with this have not been impressive. The asynchrony between nuclear and cytoplasmic maturation at the cellular level could explain the suboptimal embryo quality obtained from in vitro matured oocytes. ${ }^{1}$ Several approaches have been employed to enhance the nucleo-cytoplasmic synchrony and the most recent experiments have been on CAMP inhibitors like cilostamide that promote cytoplasmic maturation while maintaining high levels of intracellular CAMP levels keeping the nuclear maturation at check. . $^{2,3}$

The use of cAMP inhibitors thus is postulated to enhance the embryo quality but it does not give an insight into the selection of best quality embryos for transfer. The identification of the embryo with the highest implantation potential is one of the greatest challenges in the field of reproductive medicine even today. In the current era, embryo selection holds the key to make ART practice safer for these patients, as we are inching closer toward elective single embryo transfer globally. The techniques of embryo selection have evolved ever since the inception of IVF. The initial and the most commonly used techniques were based on the analysis of the embryo morphology. Embryo selection is commonly performed based on the appearance of multiple unique morphological characteristics at one or several stages of preimplantation development. ${ }^{4}$ Across the world, different embryo
1,3,5,7Department of Reproductive Medicine, Milann Fertility Centre, Bengaluru, Karnataka, India

${ }^{2,4}$ Department of Obstetrics and Gynaecology, KS Hegde Medical Academy, Mangaluru, Karnataka, India

${ }^{6}$ Department of Embryology, Garbhagudi IVF Center, Bengaluru, Karnataka, India

Corresponding Author: Prasanna Kumar Shetty, Department of Obstetrics and Gynaecology, KS Hegde Medical Academy, Mangaluru, Karnataka, India, e-mail: prasanna.kumar.shetty@gmail.com

How to cite this article: Cheruveetil MA, Shetty PK, Rao KA, et al. Impact of Prematuration Culture on Zygote Morphology in In Vitro Maturation: An Early Clue to Embryo Competence. Int J Infertil Fetal Med 2022;13(1):5-14.

Source of support: Nil

Conflict of interest: None

scoring systems have evolved over time. Despite this, the selection of the best embryo with the maximum implantation potential is still far from reality and has resulted in a strong drive for finding alternative selection methods.

Fertilization encompasses several morphological changes; the prominent one being the appearance of the male and the female pronuclei (PN) in the periphery of the oocyte, early in the process (as early as 6 hours after insemination), and the rotation of the pronucleus into the center by approximately $16-18$ hours postinsemination, or $56-58 \mathrm{~h}$ post-hCG. ${ }^{5-7}$ The equality and alignment of nucleolar precursor bodies (NPB) is the most crucial

(c) The Author(s). 2022 Open Access This article is distributed under the terms of the Creative Commons Attribution 4.0 International License (https://creativecommons. org/licenses/by-nc/4.0/), which permits unrestricted use, distribution, and non-commercial reproduction in any medium, provided you give appropriate credit to the original author(s) and the source, provide a link to the Creative Commons license, and indicate if changes were made. The Creative Commons Public Domain Dedication waiver (http://creativecommons.org/publicdomain/zero/1.0/) applies to the data made available in this article, unless otherwise stated. 
morphological change during fertilization. The NPBs are part of the nucleoli and are the site of ribosomal RNA ( $r R N A$ ) production where all proteins are constructed. ${ }^{8}$ These sites are referred to as the nucleolar organizing regions (NORs). There are only five NOR-bearing chromosomes, in other words, the heterochromatic chromosomes: $13,14,15,21$, and $22 .^{9}$

Oocyte maturation involving cytoplasmic maturation brings about transcriptional activity in each pronucleus leading to the origin of NPBs. ${ }^{10,11}$ The advent of NPB is time dependent and with time, they migrate and merge into the nucleoli. NPBs are sites of rRNA synthesis and are necessary for translation processes ideally activating the embryonic genome. ${ }^{12}$ Therefore, the precise timing and succession of these events are of paramount importance.

The presence of chromosomal abnormalities is the primary cause of failed implantations and spontaneous miscarriages among embryos derived from stimulated cycles. There can be differences regarding the pattern of pronuclear morphology, pertaining to the constitution of the conceptus chromosomes. ${ }^{13-15}$ Thus, the pronuclear and nucleoli morphology has been included in the scoring system as the association between the patterns observed and can be used to predict the embryo development and its corresponding implantation potential. ${ }^{16-18}$

In attempts to improve the developmental competence of oocytes by controlling nuclear and cytoplasmic maturation through temporary inhibition or attenuation of meiotic progress has been applied in different mammals with more or less success. Thus, the present study aims to corroborate whether cilostamide employed for synchronizing nuclear and cytoplasmic maturation has an impact on the zygote morphology with respect to pronuclear alignment, size, number, equality, and distribution of nucleoli, and the presence or absence of cytoplasmic halos. This is the first data set describing the correlation of morphology of the pronuclear zygotes with and without the advent of cilostamide.

\section{Materials and Methods}

\section{Ethical Approval}

The study was conducted at a tertiary care reproductive medicine unit in India. It was approved by the Institutional Review Board and the Nitte Central Ethics Committee NU/CEC/2018/0191 NU/CEC/2020/2069. Informed consents were obtained from patients prior to the laboratory access of oocytes and sperms.

\section{Study Population}

The present study comprised of 57 zygotes obtained from the in vitro matured oocytes of 63 patients aged between 25 and 35 years, who underwent controlled ovarian stimulation (COS) for IVF/ICSI from April 2018 to March 2020. The patients with a minimum of four germinal vesicles were included in the study Figure 1.

\section{Stimulation Protocol}

COS was started on Day 2/3 of menstrual cycle after a baseline transvaginal ultrasound scan (Voluson $\mathrm{P} 6$ with $4-8 \mathrm{MHz}$ vaginal probe) and hormonal assessment [Estradiol (E2), Progesterone (P4), Luteinizing hormone (LH) if deemed necessary]. Gonadotropins included either recombinant Follicle stimulating hormone, [Recagon, Organon, Oss, The Netherlands; GonalF, Merck Serono S.p.A, Modugno (Bari), Italy] or human menopausal gonadotropin (Menopur; Ferring Leciva, as, Czech Republic). The starting dose was calculated based on age, body mass index and antral follicle count Figures 2 and 3. It ranged between 112.5 and 187.5 IU daily for 4 days. The ovarian response to stimulation was monitored on the fifth day of stimulation with transvaginal ultrasound scan and serum E2, LH, and progesterone measurements and the dose of gonadotropins was adjusted accordingly. A gonadotrophin-releasing hormone antagonist (Cetrotide, KGaA, Darmstadt, Germany) $0.25 \mathrm{mg}$ was given daily subcutaneously based on a flexible antagonist protocol when the dominant follicle was $\geq 12-14 \mathrm{~mm}$ in diameter or the serum E2 level was $>350-400 \mathrm{pg} / \mathrm{mL}$ until the day of trigger. The triggering was given when at least three follicles

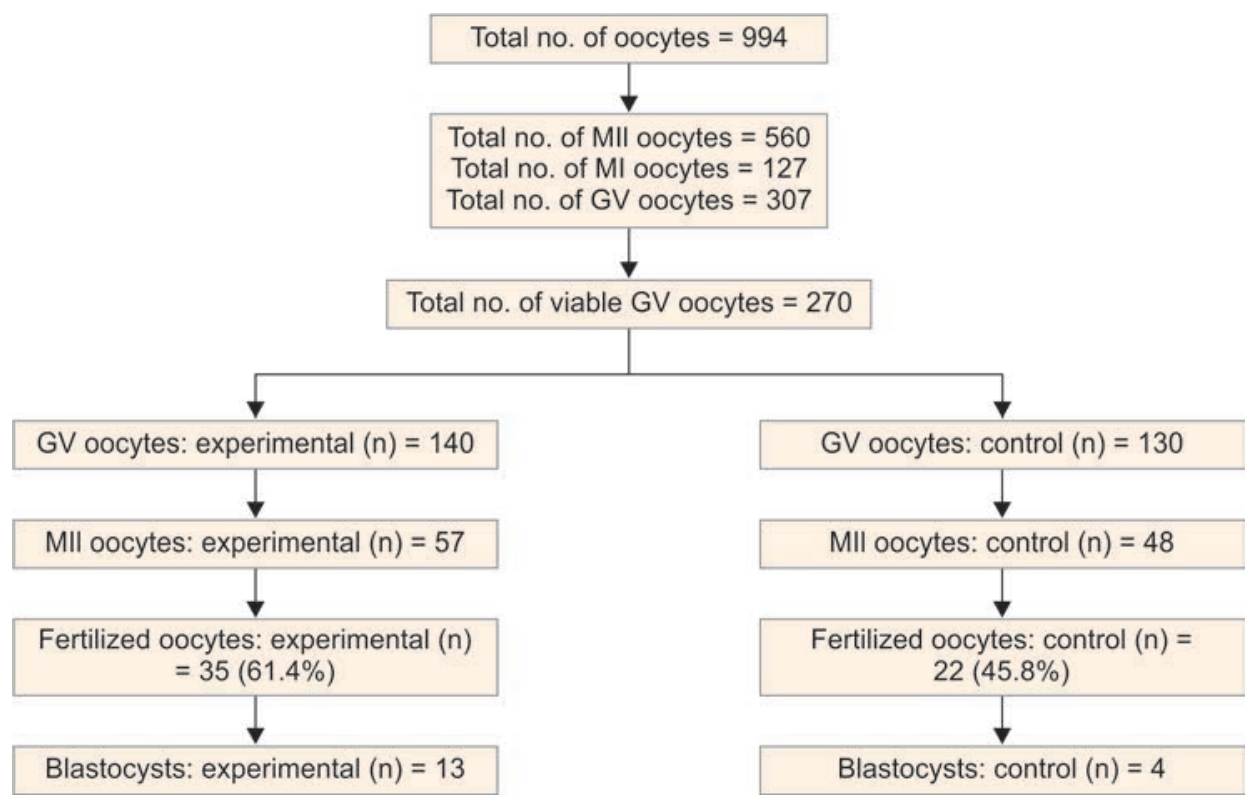

Fig. 1: Distribution of oocytes retrieved and subsequent fertilization and embryo development MI, Meiosis I; MII, Meiosis II; GV, Germinal vesicle 


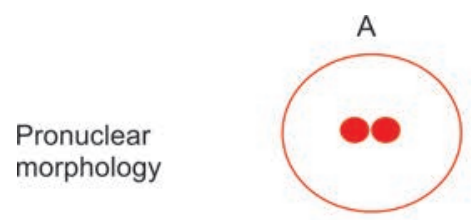

Centralized Juxtaposed



Non-centralized Juxtaposed

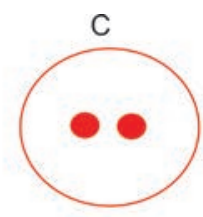

Centralized nonjuxtaposed

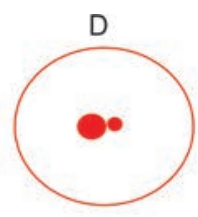

Different sizes

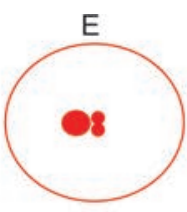

Fragmented

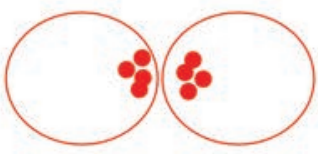

Large-size aligned
2

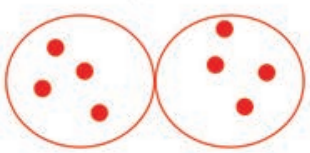

Large-size scattered

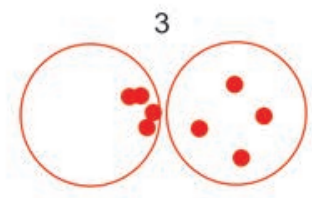

Large-size 1 pronucleus aligned

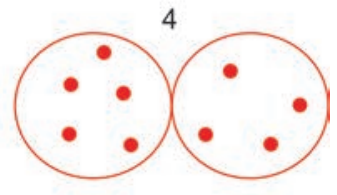

Small-size 1 scattered

Polar body alignment

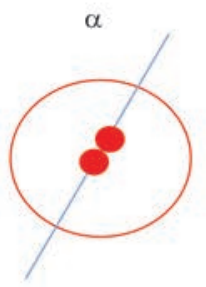

Longitudinal

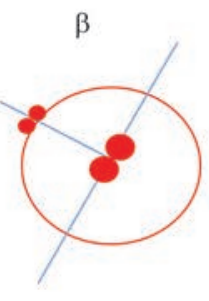

Perpendicular

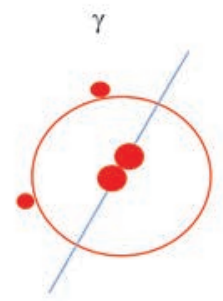

Others

Fig. 2: Depicts the scoring system performed by Gianaroli et al., which comprises pronuclear morphology (A-E), nucleolar morphology (1-4), and polar body alignment $(a, \beta, \gamma)$

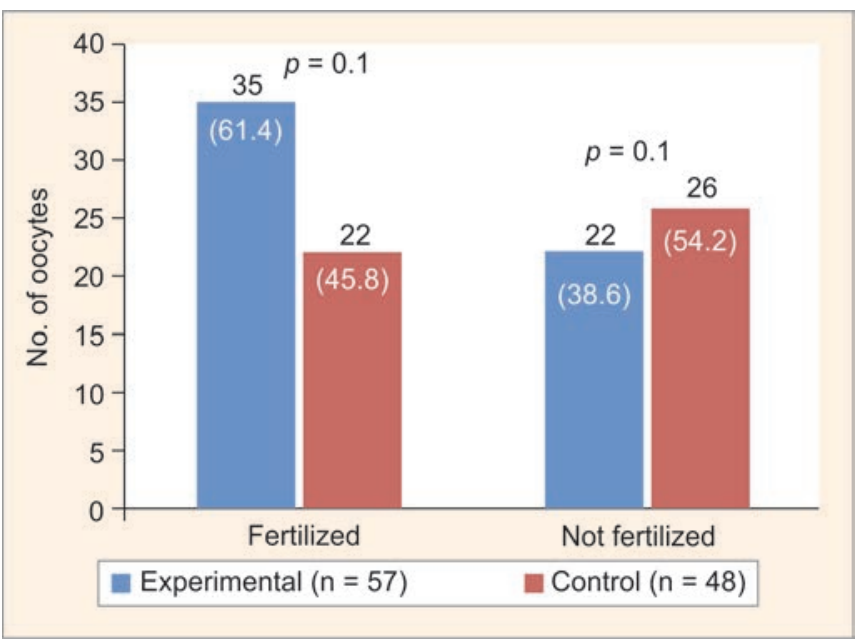

Fig. 3: Fertilization status of pronuclear zygotes developed from GV converted MII oocytes subjected to prematuration culture with cilostamide $(1 \mu \mathrm{M})$ followed by IVM culture

reached $\geq 17 \mathrm{~mm}$ in diameter. Oocytes were aspirated transvaginally 35-36 $\mathrm{h}$ post-triggering under intravenous sedation and ultrasound guidance. A single lumen oocyte retrieval needle (Vitrolife Sweden AB V.Frölunda, Sweden) was used.

\section{Laboratory Protocol}

\section{Preparation of Cilostamide (PDE3-I)}

The phosphodiesterase 3 inhibitor - cilostamide (Cayman Chemicals) was employed for prematuration culture. The stock solution was made by dissolving crystalline cilostamide in dimethyl sulfoxide. A final concentration of $1 \mu \mathrm{M}$ cilostamide was added to the prematuration culture with the intention of achieving efficient meiotic inhibition and maximum reversibility of inhibition.

\section{Experimental Setup}

\section{Prematuration Culture}

The denuded GV-stage oocytes of each patient were distributed into two groups and subjected to two different cultural conditions, in other words, the experimental and the control groups, and cultured for 6 hours. All immature oocytes were cultured individually in $25 \mu \mathrm{L}$ drops of medium overlaid with oil in a humidified atmosphere at $6 \% \mathrm{CO}_{2}$ at $37^{\circ} \mathrm{C}$ temperature. The experimental group oocytes were placed in an IVM medium (Oocyte Maturation Medium; Cooper Surgical/SAGE) supplemented with $5 \mathrm{mg} / \mathrm{mL}$ Serum Protein Substitute and $\mathrm{FSH} / \mathrm{LH}$ (75 mlU/mL for each) (Serono, Mississauga, ON, Canada) supplemented with $1 \mu \mathrm{M}$ cilostamide and the oocytes cultured in media without meiotic inhibitor cilostamide were set as control. After culturing these GV-stage oocytes for 6 hours, the inhibition through cilostamide was removed by transferring them to fresh IVM medium without cilostamide and cultured further to attain maturity for a maximum of 42 hours.

The maturational status of the oocytes was examined at 26,36 and 48 hours in the experimental and control groups, respectively. Oocytes were defined as GV, metaphase I (MI) or MII based on the appearance of the first polar body or with the absence of germinal vesicle. The transition of oocytes from GV to MII begins with the visualization of an ill-defined nuclear envelope (GVBD) to where 
neither a GV nor a PB were present (MI) and finally converts into MIl oocytes with the extrusion of a PB (Fig. 4).

\section{Intracytoplasmic Sperm Injection}

ICSI was performed on the GV converted MII oocytes using morphologically normal spermatozoa. Following ICSI, these two groups (experimental and control groups) of injected oocytes were then incubated in $25 \mu \mathrm{L}$ drops of single-step medium (GTL, VitroLife Sweden AB Västra Frölunda, Sweden) for further development.

\section{Assessment of Fertilization and Embryo Development Zygote Scoring}

Pronuclear zygotes were examined under an inverted microscope equipped with Hoffman modulation optics to determine the exact number, alignment and morphology of PN, NPBs, and polar bodies precisely at $17 \pm 1$ hour after insemination with a magnification of $40 x$ objective. The time of examination was minimized in order to maintain the temperature and $\mathrm{pH}$ of the medium, which may affect subsequent embryo development. During observation, zygotes were rolled to place both polar bodies and PN in the same plane

Zygotes were then classified by the scoring system proposed by Gianaroli et al. The scoring system was based on three criteria which includes pronuclear morphology (A-E), NPBies morphology, ${ }_{1}^{1-4}$ and PB alignment $(a, \beta, \gamma)$. (A) juxtaposed and centralized, (B) juxtaposed and peripheral, (C) centralized and separated, (D) unequal in size, and (E) fragmented. In addition, NPB morphology was evaluated according to their position and number within the PN. ${ }^{1}$ Large size, aligned; ${ }^{2}$ large size, scattered in both PN; large size, aligned in one pronucleus and scattered in the other ${ }^{4}$ small size in at least one pronucleus, scattered. The orientation of the polar bodies was described in relation to the longitudinal axis of PN: (a) in the longitudinal axis; $(\beta)$ perpendicular to the longitudinal axis; $(\gamma)$ in different positions.

\section{Results}

In total, 994 oocytes were obtained from 63 patients, of which 307 were GV oocytes. Morphologically abnormal 37 GV oocytes were excluded from the study. A total of 270 viable germinal vesicle oocytes obtained were assigned into experimental and control groups. A total of $140 \mathrm{GV}$ oocytes in the experimental group were exposed to $1 \mu \mathrm{M}$ cilostamide and $130 \mathrm{GV}$ oocytes in the control group were cultured without cilostamide. The total number of MII oocytes obtained following IVM culture were 57 and 48 in the experimental group and control group, respectively (Fig. 1). Following ICSI, a total of 57 pronuclear zygotes were generated as represented in Figure 2.

The prospective investigation included a total of 57 zygotes (35 zygotes in the experimental group and 22 in the control group). The fertilization rate was higher in the experimental group, $61.4 \%$ when compared to the control group, $45.8 \%$. However, there was no statistically significant difference in the fertilization rates $(p>0.05)$ (Fig. 3).

Fertilization assessment was performed at $17 \pm 1$ hour. Among the distribution of pronuclear morphology, the experimental group showed an increased central juxtaposed position of pronucleus as compared to the control group. But the distribution was similar in both the groups $(p>0.05)$ (Table 1).

When considering the distribution of NPBs in zygote morphology, the NPB were evaluated as per Gianaroli et al. criteria based on their position and number within the PN. The large-size aligned NPBs (Group 1) were observed in $51.4 \%$ of the cilostamide treated experimental group and $22.7 \%$ in the control group. A statistically significant increase in Group 1 NPBs were observed in the experimental group than in the control group $(p=0.03)$.

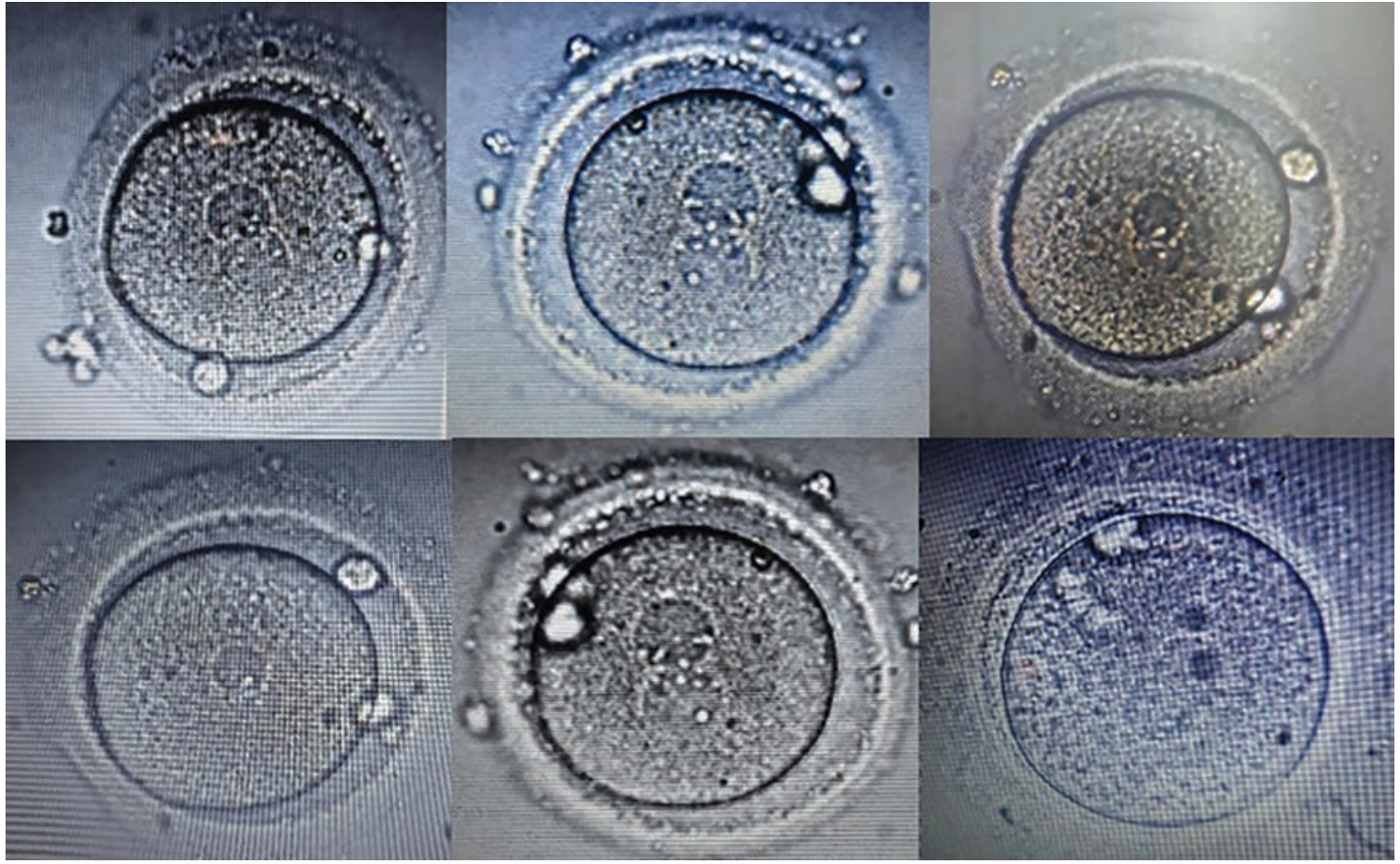

Fig. 4: Images of human pronuclear stage zygotes developed from GV oocytes subjected to prematuration culture with cilostamide (1 $\mu \mathrm{M})$ followed by in vitro maturation (IVM) culture 
Table 1: Assessment of fertilization status of GV converted MII oocytes (26, 36 and 48 hours)

\begin{tabular}{|c|c|c|c|c|}
\hline SI. No. & Assessment of fertilization & Experimental $(n=61.4 \%)$ & Control $(n=45.8 \%)$ & $p$-value \\
\hline \multirow[t]{5}{*}{1} & Position of pronucleus at $17 \pm 1$ & & & \\
\hline & Central juxtaposed & $27(77.1)$ & $12(54.5)$ & 0.07 \\
\hline & Eccentric juxtaposed & $5(14.3)$ & $7(31.8)$ & 0.1 \\
\hline & Central nonjuxtaposed & $2(5.7)$ & $2(9.1)$ & 0.6 \\
\hline & Different sizes & $1(2.9)$ & $1(4.5)$ & 0.7 \\
\hline \multirow[t]{5}{*}{2} & NPBs morphology & & & \\
\hline & Group 1 & $18(51.4)$ & $5(22.7)$ & $0.03^{*}$ \\
\hline & Group 2 & $12(34.3)$ & $7(31.8)$ & 0.8 \\
\hline & Group 3 & $4(11.4)$ & $8(36.4)$ & $0.02^{*}$ \\
\hline & Group 4 & $1(2.9)$ & $2(9.1)$ & 0.3 \\
\hline \multirow[t]{4}{*}{3} & Polar body alignment & & & \\
\hline & $a$ & $23(65.71)$ & $8(36.4)$ & $0.03^{*}$ \\
\hline & $\beta$ & $9(25.71)$ & $10(45.5)$ & 0.12 \\
\hline & $\gamma$ & $3(8.58)$ & $4(18.1)$ & 0.29 \\
\hline \multirow[t]{3}{*}{4} & Cytoplasmic halo & & & \\
\hline & Present & $21(60)$ & $10(45.5)$ & 0.2 \\
\hline & Absent & $14(40)$ & $12(54.5)$ & 0.2 \\
\hline \multirow[t]{3}{*}{5} & No. of pronucleus & & & \\
\hline & $2 \mathrm{PN}$ & $35(100)$ & $21(95.5)$ & 0.7 \\
\hline & $3 \mathrm{PN}$ & -- & $1(4.5)$ & 0.7 \\
\hline
\end{tabular}

NPBs, nucleolar precursor bodies; nucleolar precursor bodies morphology was evaluated according to their position and number within the pronuclei

(1) large size, aligned;

(2) large size, scattered in both pronuclei;

(3) large size, aligned in one pronucleus and scattered in the other;

(4) small size in at least one pronucleus, scattered. The orientation of polar bodies was described in relation to the longitudinal axis of pronuclei: (a) in the longitudinal axis; $(\beta)$ perpendicular to the longitudinal axis; $(\gamma)$ in different positions. Statistical test-Chi-square test; $p<0.05$ is considered statistically significant

Whereas, Group 3 NPBs were significantly higher in the control group than in the experimental group $(p=0.02)$ (Table 1).

Based on the distribution of polar bodies in relation to the axis of the PN, the alignment of a type PB was significantly higher in the experimental group (65.7\%) than in the control group (36.4\%) ( $p=0.03)$. But the distribution of $\beta$ and $\gamma$ cohort was similar among the experimental and control groups $(p>0.05)$ (Table 1).

Furthermore, the present study showed that the presence of cytoplasmic halos and the number of pronuclear zygotes were similar between experimental and control groups $(p>0.05)$.

During the fertilization assessment of GV converted MII oocytes at 26 hour, the central and the eccentric juxtaposed positions of PN was observed. However, the distribution of these positions was similar in both the groups.

In addition, the distribution of Group 1 NPBs was significantly higher in the experimental group than in the control group $(p=0.03)$. There was no difference in the distribution of PB alignment, cytoplasmic halo, and the number of pronucleus among the experimental and control groups (Table 2).

Upon fertilization of GV converted MII oocytes obtained at 36 hour of IVM culture, a statistically significant increase in Group 3 NPBs was observed in the control group as compared to the experimental group $(p=0.04)$. The alignment of a type PB was significantly higher in the experimental group (70.6\%) than in the control group (27.2\%) ( $p=0.27)$. The distribution of other parameters was similar among the experimental and control groups (Table 3) (Fig. 4).

In the 48th hour, the present study showed the proportion of central and eccentric juxtaposed position, Group 2 NPBs, $\beta$ type PB alignment, was comparatively higher in the experimental group than in the control group. The number of PN among the experimental and control groups were similar. However, there was no statistically significant difference between the two groups ( $p>0.05$ ) (Table 4).

In the present study the presence of cytoplasmic halos was evaluated in the fertilized oocytes. A positive halo effect was observed in 31 zygotes, and the absence of cytoplasmic halo was observed in 26 zygotes. The proportion of cytoplasmic halo was higher in the experimental group in 26 and 36 hours as compared to the control group, whereas in 48 hours the distribution was similar. However, there was no statistically significant difference between the experimental and control groups $(p>0.05)$.

\section{Discussion}

An oocyte undergoes several events during development leading to its maturation, of which, it is crucial that both nuclear and cytoplasmic maturation occur harmoniously to prepare the oocyte for resumption of meiosis and to support the events of fertilization. A fertilized oocyte is activated by the entry of spermatozoa into 
Table 2: Assessment of fertilization rate of GV converted MII oocytes, cultured in IVM media for 26 hours among experimental group and control group

\begin{tabular}{|c|c|c|c|c|}
\hline SI. No. & Assessment of fertilization & Experimental $(n=3)$ & Control $(n=9)$ & $p$-value \\
\hline \multirow[t]{3}{*}{1} & Position of pronucleus at $17 \pm 1$ & & & \\
\hline & Central juxtaposed & $11(84.6)$ & $5(55.6)$ & 0.09 \\
\hline & Eccentric juxtaposed & $2(15.4)$ & $4(44.4)$ & 0.09 \\
\hline \multirow[t]{4}{*}{2} & NPBs morphology & & & \\
\hline & Group 1 & $9(69.2)$ & $2(22.2)$ & $0.03^{*}$ \\
\hline & Group 2 & $2(15.4)$ & $3(33.3)$ & 0.3 \\
\hline & Group 3 & $2(15.4)$ & $4(44.5)$ & 0.14 \\
\hline \multirow[t]{4}{*}{3} & Polar body alignment & & & \\
\hline & $a$ & $11(84.6)$ & $5(55.6)$ & 0.14 \\
\hline & $\beta$ & $2(15.4)$ & $4(44.4)$ & 0.14 \\
\hline & $\gamma$ & 0 & 0 & -- \\
\hline \multirow[t]{3}{*}{4} & Cytoplasmic halo & & & \\
\hline & Present & $9(69.2)$ & $4(44.4)$ & 0.2 \\
\hline & Absent & $4(30.8)$ & $5(55.6)$ & 0.2 \\
\hline \multirow[t]{2}{*}{5} & Pronucleus & & & \\
\hline & 2 & $13(100)$ & $9(100)$ & -- \\
\hline
\end{tabular}

NPBs, nucleolus precursor bodies; IVM, In vitro maturation; Statistical test-Chi-square test; $p<0.05$ is considered statistically significant

Table 3: Assessment of fertilization rate of GV converted MII oocytes, cultured in IVM media for 36 hours among experimental group and control group

\begin{tabular}{|c|c|c|c|c|}
\hline Sl. No. & Assessment of fertilization & Experimental $(n=17)$ & Control $(n=11)$ & $p$-value \\
\hline \multirow[t]{5}{*}{1} & Position of pronucleus & & & \\
\hline & Central juxtaposed & $14(82.4)$ & $6(54.5)$ & 0.1 \\
\hline & Central nonjuxtaposed & $1(5.9)$ & $2(18.2)$ & 0.3 \\
\hline & Eccentric juxtaposed & $1(5.9)$ & $3(27.3)$ & 0.1 \\
\hline & Different size & $1(5.9)$ & $0(0)$ & 0.4 \\
\hline \multirow[t]{5}{*}{2} & NPBs morphology & & & \\
\hline & Group 1 & $8(47.1)$ & $3(27.3)$ & 0.3 \\
\hline & Group 2 & $7(41.2)$ & $3(27.3)$ & 0.4 \\
\hline & Group 3 & $1(5.9)$ & $4(36.4)$ & $0.04^{*}$ \\
\hline & Group 4 & $1(5.9)$ & $1(9.1)$ & 0.7 \\
\hline \multirow[t]{4}{*}{3} & Polar body alignment & & & \\
\hline & $a$ & $12(70.6)$ & $3(27.2)$ & $0.02^{*}$ \\
\hline & $\beta$ & $5(29.4)$ & $6(54.6)$ & 0.1 \\
\hline & Y & 0 & $2(18.2)$ & 0.07 \\
\hline \multirow[t]{3}{*}{4} & Cytoplasmic halo & & & \\
\hline & Present & $10(58.8)$ & $6(54.6)$ & 0.8 \\
\hline & Absent & $7(41.2)$ & $5(45.5)$ & 0.8 \\
\hline \multirow[t]{2}{*}{5} & Pronucleus & & & \\
\hline & 2 & $17(100)$ & $11(100)$ & -- \\
\hline
\end{tabular}

NPBs, nucleolus precursor bodies; IVM, In vitro maturation; Statistical test-Chi-square test; $p<0.05$ is considered statistically significant

it, which triggers a series of events essential for enhancing the embryo competence. An asynchrony in the succession of these events can cause fertilization failure and uneven divisions. Hence, assessing the morphology of fertilized oocytes can provide an insight into the embryo development and its implantation potential. ${ }^{19-22}$ The data presented in this paper reveals that IVM culture with PDE3 I has several aspects leading to the development of competent pronuclear zygotes that can generate good quality embryos with increased implantation potential. One aspect being the position of PN and the number, equality, and alignment of 
Table 4: Assessment of fertilization rate of GV converted MII oocytes, cultured in IVM media for 48 hours among experimental group and control group

\begin{tabular}{|c|c|c|c|c|}
\hline Sl. No. & Assessment of fertilization & Experimental $(n=5)$ & Control $(n=2)$ & $p$-value \\
\hline \multirow[t]{5}{*}{1} & Position of pronucleus & & & \\
\hline & Central juxtaposed & $2(40)$ & $1(50)$ & 0.8 \\
\hline & Central nonjuxtaposed & $1(20)$ & $0(0)$ & 0.5 \\
\hline & Eccentric juxtaposed & $2(40)$ & $0(0)$ & 0.3 \\
\hline & Different size & $0(0)$ & $1(50)$ & 0.1 \\
\hline \multirow[t]{5}{*}{2} & NPBs & & & \\
\hline & Group 1 & $1(20)$ & $0(0)$ & 0.5 \\
\hline & Group 2 & $3(60)$ & $1(50)$ & 0.8 \\
\hline & Group 3 & $1(20)$ & $0(0)$ & 0.5 \\
\hline & Group 4 & $0(0)$ & $1(50)$ & 0.1 \\
\hline \multirow[t]{4}{*}{3} & Polar body alignment & & & \\
\hline & $a$ & 0 & 0 & -- \\
\hline & $\beta$ & $2(40)$ & 0 & 0.3 \\
\hline & Y & $3(60)$ & $2(100)$ & 0.3 \\
\hline \multirow[t]{3}{*}{4} & Cytoplasmic halo & & & \\
\hline & Present & $2(40)$ & $0(0)$ & 0.3 \\
\hline & Absent & $3(60)$ & $2(100)$ & 0.3 \\
\hline \multirow[t]{3}{*}{5} & Pronucleus & & & \\
\hline & 3 & -- & $1(50)$ & 0.1 \\
\hline & 2 & $5(100)$ & $1(50)$ & 0.1 \\
\hline
\end{tabular}

NPBs, Nucleolus precursor bodies; IVM, In vitro maturation; Statistical test-Chi-square test; $p<0.05$ is considered statistically significant

NPBs were better among the cilostamide group in comparison to the control group showing normal development which is an essential event in any mitotic cell. Additionally, an uneven number and pattern of NPBs results in abnormal cell cycles which may lead to slower and poorer development. ${ }^{23}$

Microtubule organizing centers of the human sperm play a crucial role in the formation of polar axes at syngamy by controlling the plane of the first mitotic division thereby arriving at the direct apposition of two PN. ${ }^{24-26}$ Chromatin in both of the PN begins to polarize and then rotates to face the other pronucleus at apposition. ${ }^{27}$ Approximately $16-18$ hours after insemination, PN are in close proximity and appear to touch. Stringent temperature control is crucial during this phase since spindle disassembly and chromosome dispersal occur at varying temperatures, as have been reported at $35^{\circ} \mathrm{C} .{ }^{28,29}$ The failure to progress to apposition and syngamy could occur due to interferences in the mechanisms involved. However, majority of them depend on sperm centrosome organization The presence of abnormal pronuclear patterns could result from the presence of oocyte cytoplasmic immaturity or sperm decondensation defects. ${ }^{30,31}$ Therefore, in our study sperms with normal morphology have only been incorporated taking into account these details. Also, as a potential solution to circumvent the above-mentioned unfortunate event of cytoplasmic immaturity, we have used PDE3 inhibitor cilostamide to manifest the beneficial effect of prematuration culture on the developmental competence of human GV-stage oocytes matured in vitro.

The results of the observations made at $17 \pm 1$ hour on 57 fertilized oocytes were allocated to four main configurations (Table 1). The first morphological criterion taken into account for zygote evaluation is the position and appearance of the PN.
Table 1 criteria 1 shows the position and extent of separation of PN within the ooplasm. According to Gianaroli et al., the pronuclear morphology were of five types. ${ }^{32}$ However, in our study only four types have been observed, the fragmented type was not observed in the study. The occurrence of unequal pronucleus sizes or distances between the PN has been linked to suboptimal embryo quality, lower developmental rates and higher multinucleation rates in several studies..$^{17,33,34}$ These are in agreement with our present study which show large differences in position and size of PN in pattern C (central nonjuxtaposed) and pattern D (different size). According to previous researches, these configurations were often found to be associated with chromosomal abnormalities. ${ }^{35}$ The pattern $A$ and $B$ however, show positive results during development. Where, central (pattern A) and eccentric (pattern B) juxtaposed PN show 77.1 and $14.3 \%$ in the experimental group and 54.5 and $31.8 \%$ in the control group. Thus, zygotes that show juxtaposed PN must be prioritized over the others.

The second morphological criterion for zygote evaluation was based on the appearance of nucleoli (NPB-NPB) within the PN. NPBs majorly serve as an anlage for the development of active ribosome synthesizing nucleoli. The NPBs have multiple functions, of which organizing and regulating major and minor satellite repeats in the nuclei which is crucial.

According to a study by Fulka et al., mouse embryos derived from enucleated oocytes showed rRNA syntheses and occurrence of pre-rRNA processes, but did not transcribe the major and minor satellite repeated sequences necessary to rearrange heterochromatin around the centromere for early development of the mouse. ${ }^{36,37}$ Thus, the NPBs should have at least a few proteins associated with pericentric heterochromatin remodeling occurring 
during the first two phases of cell development. It also causes abnormal DAXX deposition (an H3.3 histone chaperone) ultimately resulting in developmental disabilities of the embryo. ${ }^{38,39}$ Taken together, it could be reasoned that NPBs may contain maternally inherited RNA and proteins associated with epigenetic mechanisms which allow proper gene expression during development. The absence of NPBs thus results in an abnormal remodeling of the satellite sequences.

A study by Gianaroli et al. shows that chromosomal analysis of pronuclear zygotes indicated a significant difference between them, with a high percentage of euploidy in embryos that developed from zygotes with large-sized nucleoli, while small-sized scattered nucleoli resulted in abnormal embryos. The results of the observations carried out on 57 fertilized oocytes at $17 \pm 1$ hour after insemination were divided into four major constructs: (Fig. 2). Our results determine that, most of the zygotes in the experimental group showed Group 1 and Group 2 NPBs (51.4 and 34.3\%) whereas the distribution of it in the control group includes Group 1, 2, and $3(22.7,31.8$, and $36.4 \%)$, respectively.

An important finding in our study is the significant difference seen in the experimental cohort of Group 1 (51.4\%) in comparison to control group (22.7\%) ( $p$ value 0.03 ) (Table 1 ) and the least common had small-size scattered nucleoli in Group 4 (2.9\%) in the experimental cohort. This finding denotes that the cilostamide employed for cytoplasmic maturation had emanated a better NPB alignment in experimental group compared to the control group. It can also be assumed that the presence of small-sized scattered nucleoli is probably an indicator of altered development which can be attributed to the chromosomal status that cause complex abnormalities among embryos.

The third morphological criterion for zygote evaluation was based on the PB alignment. The proportion of PB alignment was higher in the a group ( 65.71 vs $36.4 \%$ ) and ( 25.71 vs $45.5 \%)$ and lower in the $\beta$ group between the cilostamide treated and nontreated group. The symmetry and polarity of the orientation of the PB has a significant effect on the quality of the embryos. ${ }^{40}$ The polar bodies are suggested to be of poorer quality when they are not situated adjacent to each other and when the angle between them is large. ${ }^{32}$ One of the reasons for the reduced quality could be attributed to the suboptimal orientation of the pronucleus, leading to cytoplasmic turbulence or uneven cleavage or fragmentation. ${ }^{41}$

An additional component of the pronuclear zygote morphology evaluation is the appearance of a cytoplasmic halo during the process of PN formation. Organelles in the cortex contract to the oocyte's center, which leaves a clear zone on the cortex..$^{23,42,43}$ Payne et al. reported that prior to the formation of male and female pronucleus, a subplasmalemmal zone of translucent cytoplasm is observed. ${ }^{44}$ In due course, this focal clearing within the cortical cytoplasm (cytoplasmic flare) often progresses to involve the entire cell cortex consequently appearing as a halo.

There were literally no detectable mitochondria in the cortical region of fertilized oocytes, which indicates that microtubule organized translocation of mitochondria and other cytoplasmic elements is responsible for causing this phenomenon. The mitochondria appear to collect in an ellipsoidal mass in the perinuclear cytoplasm. ${ }^{43}$

In a previous study, a positive correlation was found between the appearance of a cytoplasmic halo and embryo quality. ${ }^{42,43}$ Insights into the halo effect have been related to cytoplasmic movement or to the difference in the distribution of mitochondria. ${ }^{36}$ ATP and calcium mobilization are thought to affect cell cycle regulation in zygotes by clustering mitochondria to perinuclear regions, but the physiological significance of mitochondrial redistribution in human zygotes is unclear. ${ }^{43,45-48}$ Moreover, the redistribution of mitochondria near the nuclei would allow immature mitochondria, such as those in zygotes, to mature on their own, assuming some input from the nucleus is required. ${ }^{47,49}$

In summary, zygotes with closely opposed PN showing distinct perinuclear condensation and showing nucleoli aligned at the pronuclear interphase were considered to have a good implantation potential. The coalescence and remodeling of functional nucleoli has a significant impact on cell growth and its functional capacity. ${ }^{50}$

Therefore, we propose that the assessment of oocyte and pronuclear zygote morphology can act as a window for predicting the embryo development and its corresponding implantation potential.

\section{Conclusion}

The cornerstone of IVM culture is to provide of an appropriate environment for the oocytes to attain developmental competence. It has been suggested that an asynchrony between nuclear and cytoplasmic maturation could explain the reduced ability of the oocyte to support the events during fertilization. The present study using a specific inhibitor cilostamide may be a significant approach in improving the cytoplasmic maturation of immature human oocytes as the results confirm that the polarization of NPBs in both PN with the PN being similar in size and the presence of cytoplasmic halo was better in the experimental group, and therefore the embryo quality and development can be enhanced.

\section{ACKNOWLedgments}

Authors would like to thank Dr Arveen Vohra and Dr Mekhala Dwarkanath B, Milann Fertility Centre, for their critical advice on this manuscript.

\section{References}

1. Moor R, Dai $Y$, Lee $C$, et al. Oocyte maturation and embryonic failure. Hum Reprod Update 1998;4(3):223-226. DOI: 10.1093/humupd/4.3.223

2. Vanhoutte L, De Sutter P, Nogueira D, et al. Nuclear and cytoplasmic maturation of in vitro matured human oocytes after temporary nuclear arrest by phosphodiesterase 3-inhibitor. Hum Reprod 2007;22(5):1239-1246. DOI: 10.1093/humrep/dem007

3. Shu $\mathrm{Y}$, Zeng $\mathrm{H}$, Ren $\mathrm{Z}$, et al. Effects of cilostamide and forskolin on the meiotic resumption and embryonic development of immature human oocytes. Hum Reprod Oxf Engl 2008;23(3):504-513. DOI: 10.1093/humrep/dem344

4. Ebner T, Moser M, Sommergruber M, et al. Selection based on morphological assessment of oocytes and embryos at different stages of preimplantation development: a review. Hum Reprod Update 2003;9(3):251-262. DOI: 10.1093/humupd/dmg021

5. Scott LA, Smith S. The successful use of pronuclear embryo transfers the day following oocyte retrieval. Hum Reprod Oxf Engl 1998;13(4):1003-1013. DOI: 10.1093/humrep/13.4.1003

6. Tesarik J, Greco E. The probability of abnormal preimplantation development can be predicted by a single static observation on pronuclear stage morphology. Hum Reprod 1999;14(5):1318-1323. DOI: 10.1093/humrep/14.5.1318

7. Scott L. Classification of pronuclei and polarity of the zygote: correlations with outcome. Color Atlas Hum Assist Reprod Lab Clin Insights 2003. 
8. Leung AKL, Lamond Al.Thedynamics of the nucleolus. Crit RevEukaryot Gene Expr 2003;13(1):39-54. DOI: 10.1615/critreveukaryotgeneexpr. v13.i1.40

9. Dimitri P, Corradini N, Rossi F, et al. The paradox of functional heterochromatin. Bio Essays 2005;27(1):29-41. DOI: 10.1002/ bies. 20158

10. Tesarik J, Kopecny V. Developmental control of the human male pronucleus by ooplasmic factors. Hum Reprod 1989;4(8):962-968. DOI: 10.1093/oxfordjournals.humrep.a137021

11. Tesarik J, Kopecny V. Assembly of the nucleolar precursor bodies in human male pronuclei is correlated with an early RNA synthetic activity. Exp Cell Res1990;191(1):153-156. DOI: 10.1016/0014-4827(90)90050-k

12. Braude $\mathrm{P}$, Bolton V, Moore $\mathrm{S}$. Human gene expression first occurs between the four- and eight-cell stages of preimplantation development. Nature 1988;332(6163):459-461. DOI: 10.1038/332459a0

13. Plachot $M$, Mandelbaum J. Oocyte maturation,fertilization and embryonic growth in vitro. Br Med Bull 1990;46(3):675-694. DOI: 10.1093/oxfordjournals.bmb.a072424

14. Munné S, Lee A, Rosenwaks Z, et al. Diagnosis of major chromosome aneuploidies in human preimplantation embryos. Hum Reprod Oxf Engl 1993;8(12):2185-2191. DOI: 10.1093/oxfordjournals.humrep. a138001

15. Gianaroli L, Magli MC, Munné S, et al. Will preimplantation genetic diagnosis assist patients with a poor prognosis to achieve pregnancy? Hum Reprod 1997;12(8):1762-1767. DOI: $10.1093 /$ humrep/12.8.1762

16. Tesarik J, Junca A, Hazout A, et al. Embryos with high implantation potential after intracytoplasmic sperm injection can be recognized by a simple, non-invasive examination of pronuclear morphology. Hum Reprod 2000;15:1396-1399. doi: 10.1093/humrep/15.6.1396

17. Scott $L$, Alvero $R$, Leondires $M$, et al. The morphology of human pronuclear embryos is positively related to blastocyst development and implantation. Hum Reprod Oxf Engl 2000;15(11):2394-2403. DOI: 10.1093/humrep/15.11.2394

18. Montag M, van der Ven H. On behalf of the German pronuclear morphology study group. Evaluation of pronuclear morphology as the only selection criterion for further embryo culture and transfer: results of a prospective multicentre study. Hum Reprod 2001;16(11):2384-2389. DOI: 10.1093/humrep/16.11.2384

19. Combelles $\mathrm{CMH}$, Cekleniak NA, Racowsky $\mathrm{C}$, et al. Assessment of nuclear and cytoplasmic maturation in in-vitro matured human oocytes. Hum Reprod 2002;17(4):1006-1016. DOI: 10.1093/ humrep/17.4.1006

20. Heikinheimo O, Gibbons WE. The molecular mechanisms of oocyte maturation and early embryonic development are unveiling new insights into reproductive medicine. Mol Hum Reprod 1998;4(8):745-756. DOI: 10.1093/molehr/4.8.745

21. Trounson A, Anderiesz C, Jones G. Maturation of human oocytes in vitro and their developmental competence. Reproduction 2001;121(1):51-75. DOI: 10.1530/rep.0.1210051

22. Eppig JJ. Coordination of nuclear and cytoplasmic oocyte maturation in eutherian mammals. Reprod Fertil Dev 1996;8(4):485-489. DOI: $10.1071 / \mathrm{rd} 9960485$

23. Scott L. Pronuclear scoring as a predictor of embryo development. Reprod Biomed Online 2003;6(2):201-214. DOI: 10.1016/s1472-6483(10)61711-7

24. Schatten G. The centrosome and its mode of inheritance: the reduction of the centrosome during gametogenesis and its restoration during fertilization. Dev Biol 1994;165(2):299-335. DOI: 10.1006/dbio.1994.1256

25. Sathananthan AH, Ratnam SS, Ng SC, et al. The sperm centriole: its inheritance, replication and perpetuation in early human embryos. Hum Reprod 1996;11(2):345-356. DOI: 10.1093/humrep/11.2.345

26. Edwards RG, Beard HK. Is the success of human IVF more a matter of genetics and evolution than growing blastocysts? Hum Reprod 1999;14(1):1-4. DOI: 10.1093/humrep/14.1.1
27. Van Blerkom J, Davis P, Merriam J, et al. Nuclear and cytoplasmic dynamics of sperm penetration, pronuclear formation and microtubule organization during fertilization and early preimplantation development in the human. Hum Reprod Update 1995;1(5):429-461. DOI: 10.1093/humupd/1.5.429

28. Pickering SJ, Braude PR, Johnson MH, et al. Transient cooling to room temperature can cause irreversible disruption of the meiotic spindle in the human oocyte. Fertil Steril 1990;54(1):102-108. DOI: 10.1016/s0015-0282(16)53644-9

29. Almeida PA, Bolton VN. The effect of temperature fluctuations on the cytoskeletal organisation and chromosomal constitution of the human oocyte. Zygote Camb Engl 1995;3(4):357-365. DOI: $10.1017 / \mathrm{s} 0967199400002793$

30. Tesarik J, Kopecny V. Development of human male pronucleus: ultrastructure and timing. Gamete Res 1989;24(2):135-149. DOI: 10.1002/mrd.1120240203

31. Rienzi L, Ubaldi F, lacobelli $M$, et al. Day 3 embryo transfer with combined evaluation at the pronuclear and cleavage stages compares favourably with day 5 blastocyst transfer. Hum Reprod Oxf Engl 2002;17(7):1852-1855. DOI: 10.1093/humrep/17.7.1852

32. Gianaroli L, Magli MC, Ferraretti AP, et al. Pronuclear morphology and chromosomal abnormalities as scoring criteria for embryo selection. Fertil Steril 2003;80(2):341-349. DOI: 10.1016/s0015-0282(03)00596-x

33. Sadowy S, Tomkin G, Munné S, et al. Impaired development of zygotes with uneven pronuclear size. Zygote Camb Engl 1998;6(2):137-141. DOI: $10.1017 / \mathrm{s} 0967199498000057$

34. Zollner U, Zollner K-P, Hartl G, et al. The use of a detailed zygote score after IVF/ICSI to obtain good quality blastocysts: the German experience. Hum Reprod Oxf Engl 2002;17(5):1327-1333. DOI: 10.1093/humrep/17.5.1327

35. Munné S, Cohen J. Chromosome abnormalities in human embryos. Hum Reprod Update 1998;4(6):842-855. DOI: 10.1093/humupd/4.6.842

36. Probst AV, Okamoto I, Casanova M, et al. A strand-specific burst in transcription of pericentric satellites is required for chromocenter formation and early mouse development. Dev Cell 2010;19(4):625-638. DOI: 10.1016/j.devcel.2010.09.002

37. Casanova M, Pasternak M, El Marjou F, et al. Heterochromatin reorganization during early mouse development requires a single-stranded noncoding transcript. Cell Rep 2013;4(6):1156-1167. DOI: 10.1016/j.celrep.2013.08.015

38. Santenard A, Ziegler-Birling C, Koch M, et al. Heterochromatin formation in the mouse embryo requires critical residues of the histone variant H3.3. Nat Cell Biol 2010;12(9):853-862.

39. Aguirre-Lavin T, Adenot P, Bonnet-Garnier A, et al. 3D-FISH analysis of embryonic nuclei in mouse highlights several abrupt changes of nuclear organization during preimplantation development. BMC Dev Biol 2012;12(1):30. DOI: 10.1186/1471-213X-12-30

40. Garello C, Baker H, Rai J, et al. Pronuclear orientation, polar body placement, and embryo quality after intracytoplasmic sperm injection and in-vitro fertilization: further evidence for polarity in human oocytes? Hum Reprod Oxf Engl 1999;14(10):2588-2595. DOI: 10.1093/humrep/14.10.2588

41. Papale L, Fiorentino A, Montag M, et al. The zygote. Hum Reprod 2012;27 (Suppl 1):i22-49. DOI: 10.1093/humrep/des205

42. Ebner T, Moser M, Sommergruber M, et al. Presence, but not type or degree of extension, of a cytoplasmic halo has a significant influence on preimplantation development and implantation behaviour. Hum Reprod 2003;18(11):2406-2412. DOI: 10.1093/humrep/deg452

43. Van Blerkom J, Davis P, Mathwig V, et al. Domains of high-polarized and low-polarized mitochondria may occur in mouse and human oocytes and early embryos. Hum Reprod 2002;17(2):393-406. DOI: 10.1093/humrep/17.2.393

44. Payne D, Flaherty SP, Barry MF, et al. Preliminary observations on polar body extrusion and pronuclear formation in human oocytes using time-lapse video cinematography. Hum Reprod 1997;12(3):532-541. DOI: 10.1093/humrep/12.3.532 
45. Wu GJ, Simerly C, Zoran SS, et al. Microtubule and chromatin dynamics during fertilization and early development in rhesus monkeys, and regulation by intracellular calcium ions. Biol Reprod 1996;55(2):260-270. DOI: 10.1095/biolreprod55.2.260

46. Diaz G, Setzu MD, Zucca A, et al. Subcellular heterogeneity of mitochondrial membrane potential: relationship with organelle distribution and intercellular contacts in normal, hypoxic and apoptotic cells. J Cell Sci 1999;112 (Pt 7):1077-1084.

47. Bavister BD, Squirrell JM. Mitochondrial distribution and function in oocytes and early embryos. Hum Reprod 2000;15(Suppl 2):189-198. DOI: 10.1093/humrep/15.suppl_2.189
48. Sousa M, Barros A, Silva J, et al. Developmental changes in calcium content of ultrastructurally distinct subcellular compartments of preimplantation human embryos. Mol Hum Reprod 1997;3(2):83-90. DOI: 10.1093/molehr/3.2.83

49. Motta PM, Nottola SA, Makabe S, et al. Mitochondrial morphology in human fetal and adult female germ cells. Hum Reprod 2000;15 (Suppl 2):129-147. DOI: 10.1093/humrep/15.suppl_2.129

50. Gámiz P, Rubio C, Remohí J, et al. The effect of pronuclear morphology on early development and chromosomal abnormalities in cleavage-stage embryos. Hum Reprod 2003;18(11):2413-249. DOI: 10.1093/humrep/deg458 